Chronic Obstructive Pulmonary Diseases: Journal of the COPD Foundation

\author{
Original Research
}

\title{
Palliative Care Referral in the Chronic Obstructive Pulmonary Disease Population
}

\author{
Connie Bradley, BSN, RN ${ }^{1}$ Rebekah Martin, BSN, RN ${ }^{1}$ Channa Porter, BSN, RN ${ }^{1}$ \\ Kimberly Richardson, MSN, APRN, FNP-C ${ }^{1}$ Andrea Stress, BSN, RN ${ }^{1}$ Jenna L. Tobin, MSN, RN, FNP-C ${ }^{1}$
}

\begin{abstract}
Abbreviations: Global initiative for chronic Obstructive Lung Disease, GOLD; chronic obstructive pulmonary disease, COPD Funding Support: none

Date of Acceptance: September 3, 2020 | Published Online Date: December 8, 2020

Citation: Bradley C, Martin R, Porter C, Richardson K, Stress A, Tobin JL. Palliative care referral in the chronic obstructive pulmonary disease population. Chronic Obstr Pulm Dis. 2021;8(1):4-6. doi: https://doi.org/10.15326/jcopdf.2020.0195
\end{abstract}

\section{College of Nursing, Medical University of South Carolina, Charleston, South Carolina, United States}

\section{Address correspondence to:}

Jenna Tobin, MSN, RN, FNP-C

Phone: 610-761-7768

Email: jlee213@msn.com

\section{Keywords:}

COPD, palliative care, palliative care referral, timing for palliative care referral

\section{To the Editor:}

According to the American Lung Association ${ }^{1}$ palliative care improves quality of life through symptom control and improving communication with health care providers. Palliative care is a resource available to anyone, regardless of age or stage of disease. ${ }^{2}$ Similarly, the Global initiative for chronic Obstructive Lung Disease (GOLD) ${ }^{3}$ supports the use of palliative care in patients with COPD regardless of disease stage. Despite substantial research supporting the benefits of palliative care, ${ }^{4}$ the utilization of palliative care for individuals diagnosed with COPD is limited. ${ }^{5}$ Moreover, research has indicated that patients with COPD would benefit from the initiation of palliative care at the beginning of the moderate stage (GOLD stage 2$)^{3}$ to address worsening dyspnea, emotional distress, inadequate coping mechanisms, and prognostic uncertainty. ${ }^{6}$
The GOLD guidelines ${ }^{3}$ recommend the integration of palliative care for symptom relief in advanced COPD stages. Traditional triggers, identified by providers, for palliative care referral in the COPD population include frequent hospitalizations, emotional symptoms, severe airflow obstruction, poor nutritional status, declining functional status, severe dyspnea, need for supplemental oxygen, and poor prognosis. ${ }^{6}$ Most of these symptoms are not an issue until late stage 3 and stage 4 when the patient and their caregivers have already been dealing with the disease for an extended time frame. ${ }^{6}$ However, several recent studies have shown that early intervention with palliative care improves quality of life and symptom control, decreases caregiver burden, and reduces aggressive and/or inappropriate endof-life interventions, thereby decreasing health care costs. ${ }^{7,8}$ It has been shown that coping styles directly correlate with depressive and anxiety symptoms, which in turn negatively affect lung function and quality of life. ${ }^{9}$ Those who possess poor coping skills suffer from severe emotional distress and higher mortality rates. ${ }^{9}$ Initiating early palliative care by stage 2 could improve coping strategies for both the patient and caregiver, as well as decrease distressing respiratory and emotional symptoms.

Palliative care, in conjunction with curative treatments, promotes and supports quality of life, offers relief from pain and stress, assists in matching treatment options with patient goals, 
and creates a positive perception for patients with COPD. ${ }^{10}$ A recent national survey concluded that misconceptions of palliative care persist, and many people associate it with end-of-life care and death. ${ }^{11}$ Misconceptions can be avoided through education of patients and caregivers. By utilizing the evidencebased definition of palliative care provided by the World Health Organization ${ }^{2}$ during conversations with patients and their caregivers, providers can help improve understanding (combat misunderstandings) of palliative care and facilitate a more open-minded approach when consulting palliative care services. A great educational resource for both providers and patients is Getpalliativecare.org. ${ }^{12}$ Here, you can find handouts for patients and families as well as access to a palliative care provider directory.

Palliative care promotes quality of life while concurrently meeting the patient's treatment goals. Therefore, it is the opinion of this group that the benefits of palliative care should be discussed at the time of diagnosis with a referral initiated at the beginning of GOLD stage 2. When clinicians use palliative care for the management of COPD, patients' treatments are more comprehensive by addressing physical and emotional concerns, decreasing the number of hospitalizations and emergency department visits, and providing the patient with optimal symptom control.

Sincerely, Connie Bradley

Rebekah Martin

Channa Porter

Kimberly Richardson

Andrea Stress

Jenna Tobin

\section{Acknowledgements}

The authors wish to thank Dr. Whitney Smith for her guidance in preparation of this editorial, as well as the Medical University of South Carolina Writing Center for proof-reading this submission. This letter to the editor was composed by doctoral and masters nursing students without any funding support.

\section{Declaration of Interest}

The authors have no known conflicts of interest to disclose. 


\section{References}

1. American Lung Association (ALA). Palliative care and COPD. ALA website. Updated October 2020. Accessed 2020.

https://www.lung.org/lung-health-diseases/lung-diseaselookup/ copd/treating/palliative-care-and-copd

2. World Health Organization (WHO). WHO definition of palliative care. WHO website. Published 2020. Accessed 2020. Retrieved from https://www.who.int/cancer/palliative/definition/en/

3. Global Initiative for Chronic Obstructive Lung Disease (GOLD). Global strategy for the diagnosis, management, and prevention of chronic obstructive pulmonary disease, 2020 report. GOLD website. Published December 2019. Accessed 2020. https: / goldcopd.org/wp-content/uploads/2019/12/GOLD2020-FINAL-ver1.2-03Dec19_WMV.pdf

4. Diop MS, Rudolph JL, Zimmerman KM, Richter MA, Skarf LM. Palliative care interventions for patients with heart failure: a systematic review and meta-analysis. J Palliat Med. 2017;20(1):84 92. doi: https://doi.org/10.1089/jpm.2016.0330

5. Brown C, Jecker NS, Curtis JR. Inadequate palliative care in chronic lung disease. An issue of health care inequality. Ann Amer Thorac Soc. 2016;13(3):311-316.

doi: https://doi.org/10.1513/AnnalsATS.201510-666PS

6. Iyer AS, Dionne-Odom JN, Khateeb DM, et al. A qualitative study of pulmonary and palliative care clinician perspectives on early palliative care in chronic obstructive pulmonary disease. J Palliat Med. 2020;23(4):513-526.

7. Han MK, Martinez CH, Au DH, et al. Meeting the challenge of COPD care delivery in the USA: a multi-provider perspective. Lancet Respir Med. 2016;4(6):473-526.

doi: http://dx.doi.org/10.1016/S2213-2600(16)00094-1

8. Lilly EJ, Senderovich H. Pulmonary palliative care in chronic obstructive pulmonary disease. J Crit Care. 2016;35:150-154. doi: https://doi.org/10.1016/j.jcrc.2016.05.019

9. Papava I, Oancea C, Enatescu VR, et al. The impact of coping on the somatic and mental status of patients with COPD: a crosssectional study. Int J Chron Obstr Pulmon Dis. 2016;11:1343-1351. doi: https://doi.org/10.2147/COPD.S106765

10. Meier DE, Morgan L. Key findings in the perceptions of palliative care. Center to Advance Palliative Care website. Published August 2019. Accessed 2020. https://media.capc.org/recorded-webinars/ slides/1lessAudience_Research_Webinar_Aug_8-2019_FINAL.pdf

11. Flieger SP, Chui K, Koch-Weser S. Lack of awareness and common misconceptions about palliative care among adults: insights from a national survey. J Gen Intern Med. 2020;35:2059-2064.

12. Get Palliative Care. Chronic obstructive pulmonary disease (COPD) and palliative care. Get Palliative Care website. Published 2020. Updated 2021. Accessed 2020. https://getpalliativecare.org/ whatis/disease-types/chronic-obstructive-pulmonary-diseasecopd-palliative-care/ 